\title{
Dendritic cells respond to nasopharygeal carcinoma cells through annexin A2-recognizing DC-SIGN
}

\author{
Pin-Zhir Chao ${ }^{1,2, *}$, Ming-Shium Hsieh ${ }^{1,3, *}$, Chao-Wen Cheng ${ }^{1}$, Tin-Jui Hsu ${ }^{4}$, Yun-Tien \\ Lin ${ }^{4}$, Chang-Hao Lai ${ }^{4}$, Chen-Chung Liao ${ }^{5}$, Wei-Yu Chen', Ting-Kai Leung7, Fei-Peng \\ Lee $^{8}$, Yung-Feng Lin ${ }^{4}$ and Chien-Ho Chen ${ }^{4}$ \\ ${ }^{1}$ Graduate Institute of Clinical Medicine, College of Medicine, Taipei Medical University, Taipei, Taiwan \\ 2 Department of Otolaryngology, Shuang-Ho Hospital, New Taipei, Taiwan \\ 3 Department of Orthopedics, En Chu Kong Hospital, New Taipei, Taiwan \\ 4 School of Medical Laboratory Science and Biotechnology, College of Medical Science and Technology, Taipei Medical \\ University, Taipei, Taiwan \\ ${ }^{5}$ Proteomics Research Center, National Yang-Ming University, Taipei, Taiwan \\ ${ }^{6}$ Department of Pathology, Wan Fang Hospital, Taipei, Taiwan \\ ${ }^{7}$ Department of Radiology, School of Medicine, College of Medicine, Taipei Medical University, Taipei, Taiwan \\ ${ }^{8}$ Department of Otolaryngology, Head and Neck Surgery, Wan-Fang Medical Center, Taipei, Taiwan \\ * These authors contributed equally to this work \\ Correspondence to: Yung-Feng Lin, email: yflin@tmu.edu.tw \\ Chien-Ho Chen, email: chenchho@tmu.edu.tw
}

Keywords: dendritic cell, DC-SIGN, NPC, annexin A2, IL-10, immunosuppression

Received: Septemeber $032014 \quad$ Accepted: November 06, $2014 \quad$ Published: November 06, 2014

This is an open-access article distributed under the terms of the Creative Commons Attribution License, which permits unrestricted use, distribution, and reproduction in any medium, provided the original author and source are credited.

\section{ABSTRACT}

Dendritic cells (DCs) play an essential role in immunity and are used in cancer immunotherapy. However, these cells can be tuned by tumors with immunosuppressive responses. DC-specific intercellular adhesion molecule 3-Grabbing Nonintegrin (DC-SIGN), a C-type lectin expressed on DCs, recognizes certain carbohydrate structures which can be found on cancer cells. Nasopharyngeal carcinoma (NPC) is an epithelial cell-derived malignant tumor, in which immune response remains unclear. This research is to reveal the molecular link on NPC cells that induces the immunosuppressive responses in DCs. In this article, we report identification of annexin A2 (ANXA2) on NPC cells as a ligand for DC-SIGN on DCs. N-linked mannoserich glycan on ANXA2 may mediate the interaction. ANXA2 was abundantly expressed in NPC, and knockdown of ANXA2 suppressed NPC xenograft in mice, suggesting a crucial role of ANXA2 in NPC growth. Interaction with NPC cells caused DC-SIGN activation in DCs. Consequently DC maturation and the proinflammatory interleukin (IL)-12 production were inhibited, and the immunosuppressive IL-10 production was promoted. Blockage of either DC-SIGN or ANXA2 eliminated the production of IL10 from DCs. This report suggests that suppression of ANXA2 at its expression or glycosylation on NPC may improve DC-mediated immunotherapy for the tumor.

\section{INTRODUCTION}

Dendritic cells (DCs) are essential antigenpresenting cells (APCs) recognizing pathogens and tumors. An effective response to tumors requires innate and adaptive immunity coordinated by DCs, key regulators of $\mathrm{T}$ cell-mediated immune responses. Recently the use of DCs becomes a major focus in cancer immunotherapy; however, many DC therapies resulted in limited clinical benefits [1]. DCs can polarize naïve T cells toward Th1 or Th2 pathway, which plays an important role on the way to specify immune responses [2]. Two receptor families on DCs are involved in this process. Toll-like receptors 
recognize common pathogen-associated molecules, while C-type lectins are receptors recognizing glycosylated antigens [3]. Toll-like receptors signal to promote DC maturation and induce the production of Th1-polarizing cytokines such as IL-12. In contrast, immature DCs strongly express DC-specific intercellular adhesion molecule-3-grabbing nonintegrin (DC-SIGN; CD209; UniProtKB: Q9NNX6), a kind of C-type lectins [4]. DCSIGN is expressed on myeloid, dermal and monocytederived DCs and functions through calcium-dependent carbohydrate binding [5]. The engagement of DC-SIGN by mannose- or fucose-containing oligosaccharides can lead to an altered Toll-like receptor signaling, resulting in a Th2 response $[5,6]$. DC-SIGN signaling increases IL-10 production which is critical for proper immunosuppression $[7,8]$.

Tumors can escape the immune system by interfering with DC activities including DC maturation and certain cytokine productions [9]. Glycosylation changes associated with tumors play an important role on inactivation of DCs [10]. Recent studies demonstrated that mannose- and fucose-expressing ligands on microbes induced different effects on the function of DCs [11]. Mannose-expressing ligands triggers an inflammatory response mediated by DCs, whereas fucose expressing ligands suppress the ability of DCs to produce the inflammatory cytokine such as IL-12, enhancing the production of IL-10. Other findings indicated that tumor-related glycol-forms of certain proteins such as carcinoembryonic antigen (CEA), Mac-2-binding protein (Mac-2BP) and mucin (MUC) 1 are specific ligands for DC-SIGN [12-14]. They instruct DCs to drive Th2mediated responses that, unlike Th1 responses, do not contribute to tumor eradication [15].

Nasopharyngeal carcinoma (NPC) is the most common tumor originating in the nasopharynx and differs from other head and neck cancers. It is most commonly found in East Asia and Africa with viral, dietary and genetic factors related in its causation. Recurrent NPC represents a small proportion of head-and-neck cancers with a unique set of patho-clinical characteristics [16]. Although the outcomes in patients with primary NPC have improved due to advances in radiotherapy and chemotherapy, the management of recurrent NPC remains a clinical dilemma because of an incomplete understanding of its pathologic mechanism. Alternatively, the patients may be treated with immunotherapy which is being studied intensively. Recent studies reported the presence of DCs in NPC biopsies, and DCs are often found inside the malignant cell nests, suggesting a potential DC-based immunotherapy for NPC [17-19]. In addition, a couple of DC-SIGN genotypes were found to be associated with NPC risk [20]; however, the role of DCs in NPC remains unclear.

In the present study, we identified annexin A2 (annexin II; ANXA2; UniProtKB: P07355) on NPC cells as a DC-SIGN ligand. ANXA2 can form a heterotetramer with p11 (S100A10) and associate with plasma membrane $[21,22]$. Its deregulation was found in a variety of tumors, including NPC, associated with the occurrence, invasion and metastasis of those tumors [23]. ANXA2 is a glycoprotein with at least one site of asparagine $(\mathrm{N})$-linked biantennary mannosyl residues [24].

We hypothesize that NPC cells escape from the surveillance of immune system by modifying DCSIGN ligand ANXA2, resulting in immunosuppressive cytokine production in DCs through DC-SIGN signaling. Indeed, we identified ANXA2 as a target for DC-SIGN. The glycan modification of ANXA2 on NPC cells was involved in their interaction, following which can alter DC activities toward a Th2 response.

\section{RESULTS}

\section{Maturation and cytokine production of MDDCs were altered by NPC cells}

To test the effect of NPC cells on DCs, we cultured MDDCs with TW01 NPC cells. With LPS stimulation, the MDDCs expressed a high level of the maturation marker HLA-DR, a subunit of antigen presenting complex, and DC-SIGN (Fig. 1A, left panel). Upon co-cultured with NPC cells, only a small portion of MDDCs expressed HLA-DR and DC-SIGN, suggesting an inhibitory effect of NPC cells on DC maturation (Fig. 1A, right panel). We then collected the culture media and determined the concentration of proinflammatory cytokine IL-12 and immunosuppressive cytokine IL-10 by ELISA. Within $72 \mathrm{~h}$, LPS-stimulated MDDCs produced IL-12 and IL-10 consistently with highest levels around $24^{\text {th }} \mathrm{h}$ (Fig. 1B). Under NPC-co-cultured condition, IL-12 production in the MDDCs was gradually decreased after $24 \mathrm{~h}$ (Fig. 1B, left panel), while IL-10 kept increasing afterward (Fig. 1B, right panel). These data indicated that NPC cells interfered with DC maturation and directed DC cytokine production toward immunosuppressive responses which could be mediated through DC-SIGN.

\section{NPC cells expressed DC-SIGN ligand(s)}

When NPC cells were treated with DC-SIGN recombinant protein and labelled with DC-SIGN antibody and FITC in the presence of $\mathrm{Ca}^{2+}$, the cells became fluorescent in flow cytometry (Fig. 2A). This result was not seen in the presence of EDTA, a $\mathrm{Ca}^{2+}$ chelator, or replacing DC-SIGN with IgG, indicating a specific binding of DC-SIGN on NPC cells. Consistent results were seen in the immunofluorescence staining (Fig. 2B). These data suggest that NPC cells express DC-SIGN ligand(s) on their cell membrane. 
DC-SIGN antibody and siRNA were applied to further determine the dependence of DC-SIGN for MDDC and NPC cell interactions. As shown on Fig. 3, DC-SIGN antibody was dramatically inhibited both the IL-10 productions promoted by NPC whole cells (Fig. 3A) and their membrane proteins (Fig. 3B) after $24 \mathrm{~h}$ of culture. Likewise, the siRNA completely inhibited NPC-promoted IL-10 production of MDDCs (Fig. 3C). These results demonstrate a specific interaction of NPC cells with DCs through DC-SIGN.

\section{DC-SIGN bound ANXA2 which was highly expressed in NPC}

DC-SIGN-Fc recombinant protein was used to precipitate NPC membrane proteins in the presence of $\mathrm{Ca}^{2+}$ or EDTA. On the silver-stained gel, a protein species with a molecular weight around $37 \mathrm{kDa}$ was found in the presence of $\mathrm{Ca}^{2+}$ but not EDTA (Fig. 4A). After LC/ MS/MS analyses, ANXA2 was identified as a significant interacting protein of DC-SIGN (Fig. 4B). Western blotting with DC-SIGN and ANXA2 antibodies on the same precipitates confirmed that ANXA2 from NPC cells bound DC-SIGN (Fig. 4C).

We then examined the expression pattern of ANXA2 in NPC. Immunohistochemistry data showed a higher ANXA2 level in patient's NPC than in normal nasopharyngeal tissue (Fig. 5A). High expression level of ANXA2 was also found in TW01 NPC cells (Fig. 5B). Fluorescent images of the cells showed colocalization of ANXA2 and the exogenous DC-SIGN-Fc on NPC cell surface in the presence of $\mathrm{Ca}^{2+}$. Taken together, ANXA2 interacts with DC-SIGN and may play an important role in the DC-mediated immunosuppression.

A


B
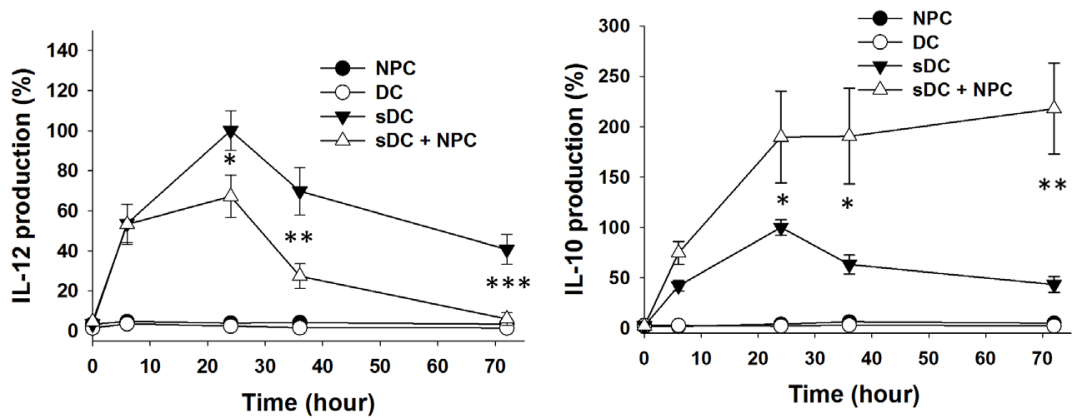

Figure 1: Maturation and cytokine production of DC co-cultured with NPC cells. A. Flow cytometry on DC showing HLADR and DC-SIGN expression. DCs without labelling (mock) were shown in black. Cells labelled with HLA-DR-FITC or DC-SIGN-FITC were shown in gray curves. Left panels, LPS-stimulated DC; right panels, LPS-stimulated DC co-cultured with NPC cells. B. IL-12 (left panel) and IL-10 (right panel) production of DC co-cultured with NPC cells in various conditions and time periods. The cytokine production levels from LPS-stimulated DC at $24 \mathrm{~h}$ were set as control (100\%). At least three sets of independent experiments were performed. * $\mathrm{p}<0.05$, $* * \mathrm{p}<0.01, * * * \mathrm{p}<0.001$. 


\section{NPC cell activities were reduced when ANXA2 was knocked down}

ANXA2 was knocked down by RNA interference to characterize the role of ANXA2 in NPC cells. Both ANXA2 shRNAs knocked down the expression at both mRNA and protein levels in NPC cells (shNPC-1 and shNPC-2) efficiently (Fig. 6A). After treated with DCSIGN-Fc recombinant protein and anti-human $\mathrm{IgG}(\mathrm{Fc}$ fragment)-FITC antibody, fluorescent NPC cells were counted in flow cytometry. The mean fluorescent intensity was reduced in both ANXA2-knocked down cell lines (Fig. 6B). The results indicated a specific interaction of ANXA2 and DC-SIGN although the reduction in shNPC-2 cells seemed not significant, which could be
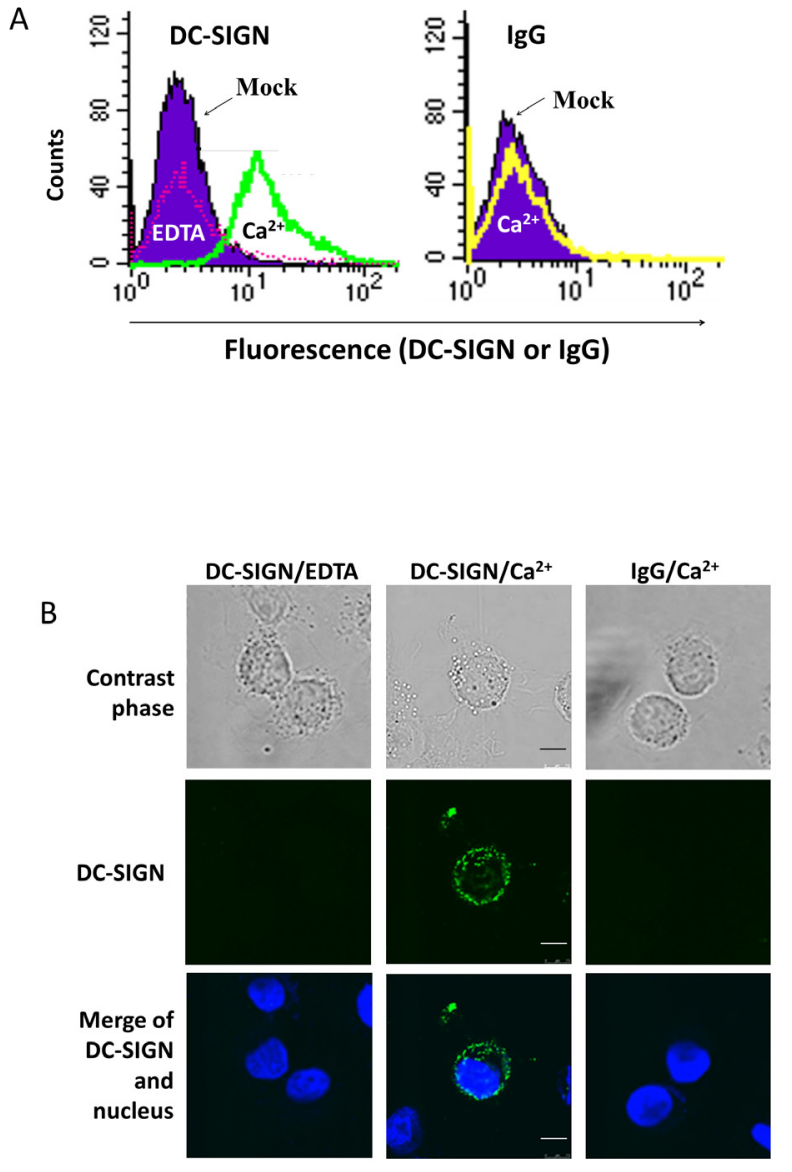

Figure 2: Expression of DC-SIGN ligand(s) on NPC cell surface. A. Flow cytometry on NPC cells treated with DC-SIGN recombinant protein and labelled with DC-SIGN antibody and FITC. NPC cells without treatment were shown in purple. The DC-SIGN-treated and -labelled NPC cells in the presence of EDTA and $\mathrm{Ca} 2+$ were shown in pink and green curves, respectively (left panel). The DC-SIGN-treated but IgGlabelled NPC cells were shown in a yellow curve (right panel). B. Confocal fluorescent images showing DC-SIGN binding on NPC cell surface. The nuclei were stained with DAPI. Three independent experiments were performed. Scale bar, $10 \mu \mathrm{m}$. due to in vitro interferences on the binding capacity of DC-SIGN-Fc. Indeed, both ANXA2-knocked down NPC cell lines were significantly decreased in promoting MDDCs to produce IL-10 (Fig. 6C). When shNPC-2 cells were used as a xenograft in mice, the tumor growth was dramatically inhibited compared to the control mice (Fig. $6 \mathrm{D})$, suggesting a potent antitumor effect of ANXA2 knockdown, which may involve a restoration from DCmediated immune suppression.

\section{Certain glycosylation pattern is required for the binding of ANXA2 by DC-SIGN}

To determine the type of glycan involved in the interaction of ANXA2 and DC-SIGN, NPC cell membrane

A
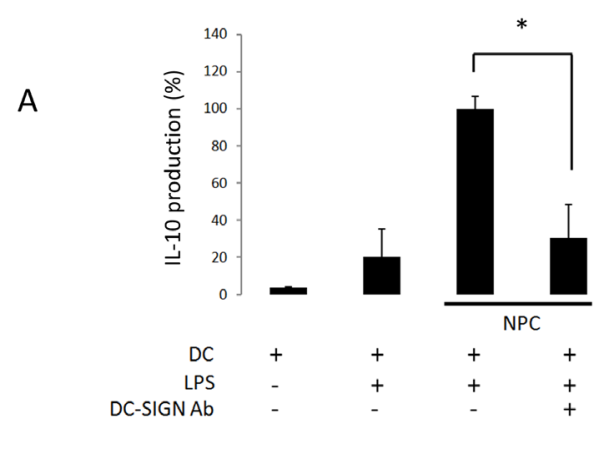

B
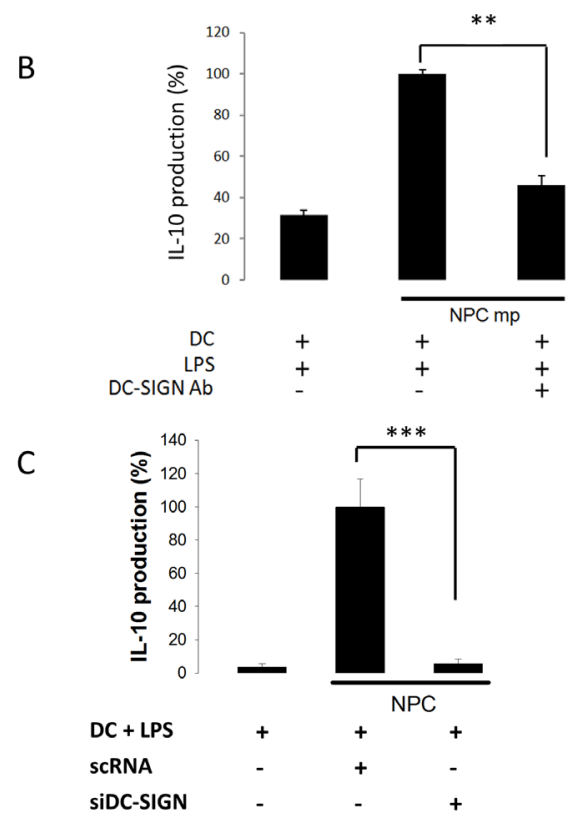

Figure 3: NPC-regulated IL-10 production of DC through DC-SIGN. A. Reduced IL-10 production from antiDC-SIGN antibody-treated DC co-cultured with NPC cells. B. Reduced IL-10 production from anti-DC-SIGN antibody-treated DC incubated with NPC membrane proteins (mp). C. Reduced IL-10 production from DC-SIGN-silenced DC co-cultured with NPC cells. The IL-10 level from LPS- and NPC-stimulated DC was set as $100 \%$ control. Three sets of each experiment were performed. $* \mathrm{p}<0.05, * * \mathrm{p}<0.01, * * * \mathrm{p}<0.001$. 
A

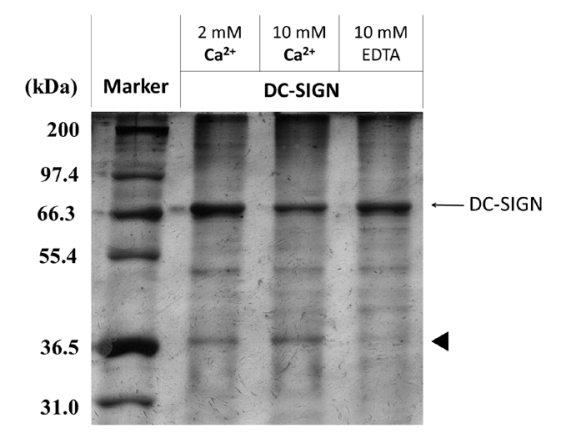

B

\begin{tabular}{|l|l|l|l|c|c|}
\hline $\begin{array}{l}\text { Accession } \\
\#\end{array}$ & $\begin{array}{l}\text { Protein } \\
\text { name }\end{array}$ & $\begin{array}{l}\text { MW } \\
(\mathrm{Da})\end{array}$ & Description & \multicolumn{2}{|c|}{ Spectrum count } \\
\cline { 3 - 6 } & $\begin{array}{l}\text { Annexin II; } \\
\text { Annexin A2; } \\
\text { ANXA2 }\end{array}$ & 38604 & $\begin{array}{l}\text { Calcium-regulated } \\
\text { membrane- } \\
\text { binding protein }\end{array}$ & 18 & 0 \\
\hline
\end{tabular}

C

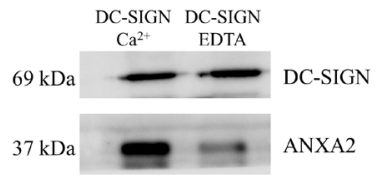

Figure 4: Immunoprecipitation of DC-SIGN-binding proteins on NPC cell membranes. NPC cell membrane proteins were incubated with human IgG-Fc-fused DC-SIGN recombinant protein at various conditions and precipitated using protein A beads. A. SDSPAGE of immunoprecipitates. Proteins were analyzed in a $12 \%$ SDS-PAGE with silver staining. Indicated proteins (arrow head) were extracted and identified by LC MS/MS. B. Identification of ANXA2 as a DC-SIGN-binding protein. C. Immunoblotting of DC-SIGN precipitates. Proteins were analyzed by immunoblotting with antibodies against DC-SIGN and ANXA2. Three independent experiments were performed.

A

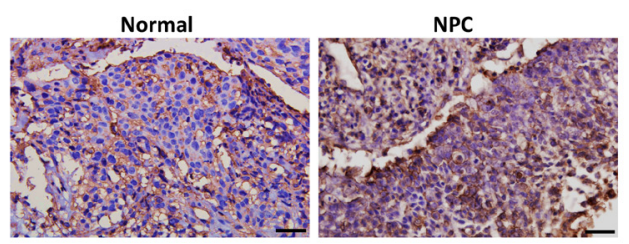

B

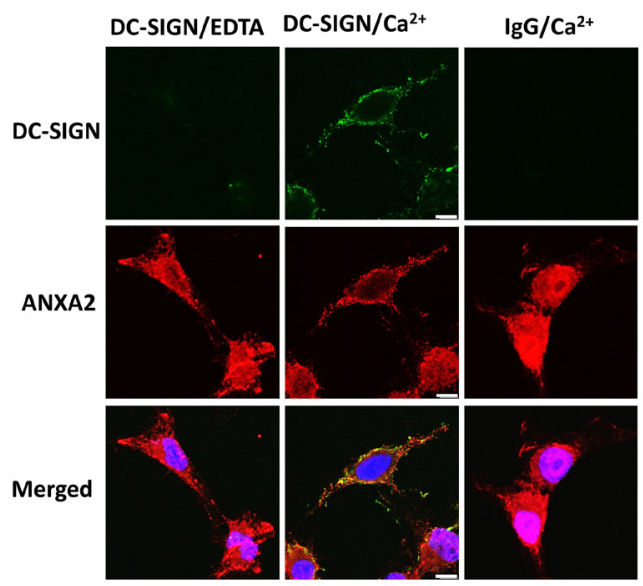

Figure 5: Expression and localization of ANXA2 in NPC. A. Immunohistochemistry of human normal and NPC tissues showing the expression of ANXA2. DAB and hematoxylin were used for the staining. Scale bar, $50 \mu \mathrm{m}$. B. Confocal fluorescent images showing the co-localization of Annexin A2 and DC-SIGN on NPC cells. Anti-Human IgG-Fc-FITC or anti-rabbit IgG-DyLight 594 antibody was used. Three sets of independent experiments were performed. Scale bar, $10 \mu \mathrm{m}$. 
proteins were treated with PNGase F, an N-glycandigesting enzyme, and then precipitated with DC-SIGNFc. As shown in Fig. 7A, DC-SIGN-Fc bound less ANXA2 with PNGase $\mathrm{F}$ treatment than that without treatment, suggesting the involvement of N-linked glycosylation on ANXA2 in NPC. Two monosaccharides, namely fucose and mannose, were then used to compete the binding of DC-SIGN-Fc on NPC cell. Flow cytometry results showed no inhibition of DC-SIGN binding on NPC cells by 20 $\mathrm{mM}$ fucose (Fig. 7B). In contrast, mannose inhibited the binding with an $\mathrm{IC}_{50}$ of $10 \mathrm{mM}$ (Fig. 7C), suggesting that mannose may constitute an important part in the glycan moiety of ANXA2 on NPC cells.

\section{DISCUSSION}

The use of DCs is a major focus in cancer immunotherapy; however, many attempts resulted in limited clinical outcomes which may be due to DC-SIGNmediated immunosuppressive responses. In this study, we identified ANXA2 on NPC cells as a ligand for DC-SIGN on DCs. Interaction of ANXA2 and DC-SIGN inhibited DC maturation and promoted immunosuppressive IL-10 production, resulting in NPC outgrowth. We therefore propose that ANXA2 may be used for target therapy on NPC and perhaps other cancers.

A

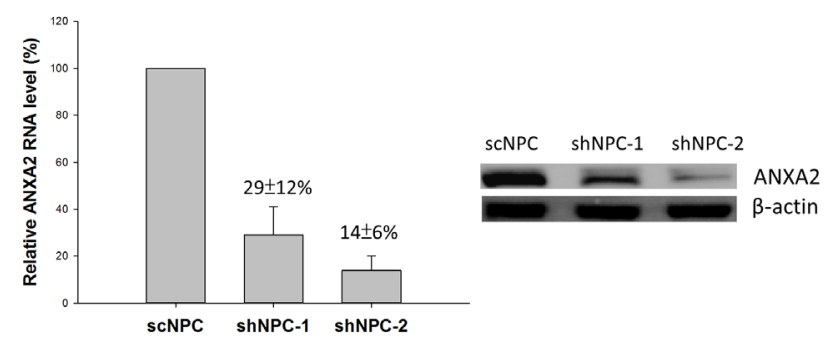

B
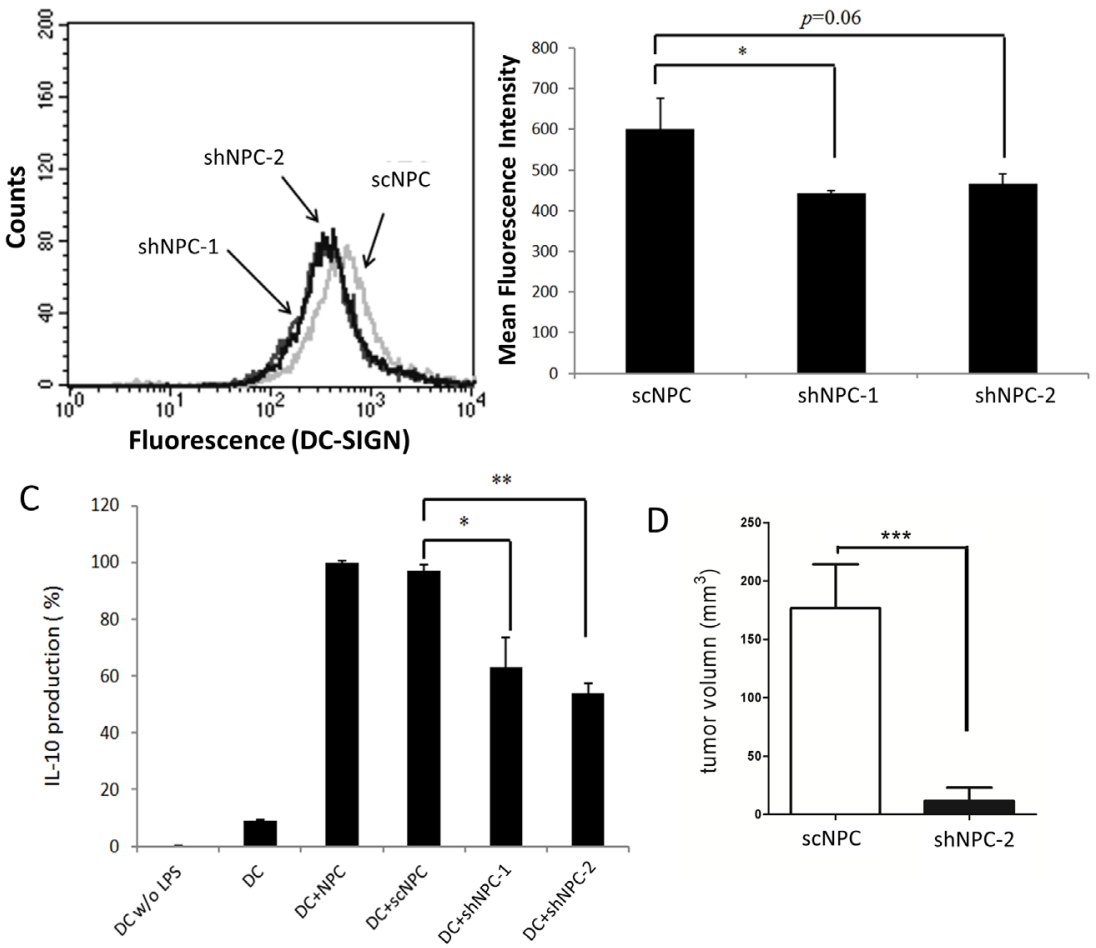

D

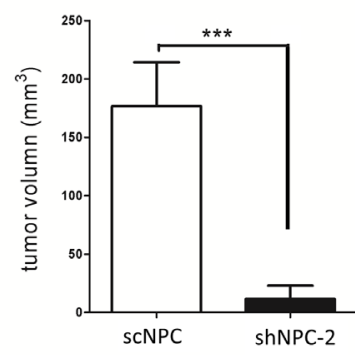

Figure 6: Reduction of NPC activities by ANXA2 knockdown. A. Knockdown of ANXA2 in NPC cells by shRNAs. The mRNA levels of NPC cells harboring a scramble shRNA (scNPC) or ANXA2 shRNA (shNPC-1 or shNPC-2) were determined by real-time PCR (left panel). The protein levels of the shRNA-harboring NPC cell lines were assayed by western blotting (right panel). B. Flow cytometry showing the reduction of DC-SIGN binding capacity of NPC cells with ANXA2 knockdown. The shRNA-harboring NPC cells were incubated with DC-SIGN-Fc recombinant protein and labelled with anti-DC-SIGN antibody for flow cytometric analysis (left panel). The statistical results were shown in the right panel. C. ELISA showing reduced IL-10 production from DC co-cultured with ANXA2-knocked down NPC cells. D. Human NPC xenografts in mice. The tumor volume on immunodeficient NSG mice hosting shNPC-2 cells was reduced comparing to those hosting scNPC. Three sets of each experiment were performed. ${ }^{*} \mathrm{p}<0.05, * * \mathrm{p}<0.01, * * * \mathrm{p}<0.001$. 
ANXA2 is a calcium-dependent, phospholipidbinding protein found on the surface of many cell types [21, 22]. The formation of ANXA2-S100A10 heterotetramer results in the association of the complex with plasma membrane. Recently it was shown that ANXA2 heterotetramer facilitates human papillomavirusinhibited maturation of Langerhans cell, another type of APCs, inducing immune suppression [25]. Indeed, ANXA2 plays a key role in immune tolerance. In the present study, we further described that DC-SIGN is an interacting partner of ANXA2, and a certain glycosylation pattern on ANXA2 is required for their interactions.

DC-SIGN recognizes certain carbohydrate structures on a variety of proteins. Recent studies of colon cancer revealed interactions of DC-SIGN and a few glycoproteins such as CEA, Mac-2BP and MUC1 on the cancer cell surface [12-14]. Their interactions with DCSIGN interfere with DC maturation and increase IL-10 production $[13,26]$, similar to the effects of ANXA2-DCSIGN interaction in the present report (Fig. 1); however, these proteins vary in the composition of glycans. ANXA2 contains N-linked glycosylation with mannose to interact with DC-SIGN (Fig. 7) that is similar to CEA, while Mac$2 \mathrm{BP}$ requires fucose to do so, and MUC1 is far different in O-linked structures.

It seems unexpectedly that ANXA2-knockdown NPC cells still bound DC-SIGN with remaining three quarters of capacity (Fig. 6B). Note that DC-SIGN can bind carbohydrate structures on other proteins. The reduction of $69-86 \%$ ANXA2 in the cells (Fig. 6A) removed nearly a quarter of DC-SIGN binding capacity, suggesting that there is about one third of DC-SIGN

A



B

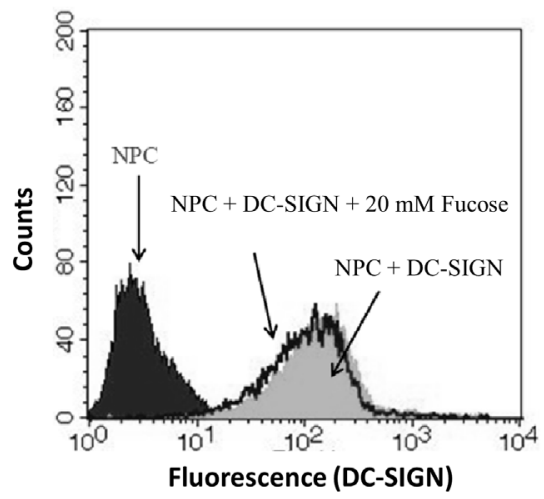

C
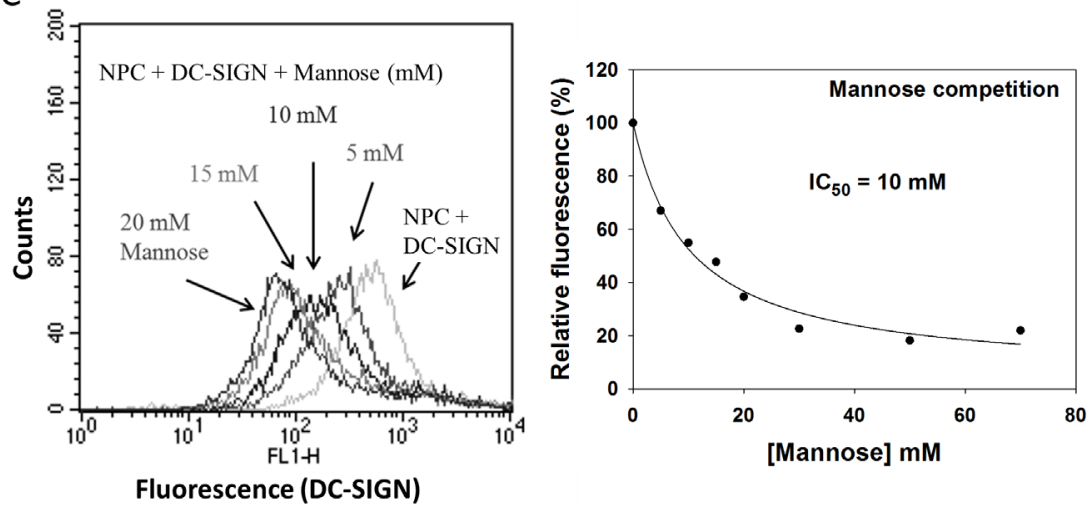

Figure 7: Involvement of glycans on NPC cells in binding DC-SIGN. A. Western blotting of DC-SIGN precipitates showing reduced ANXA2 pulldown from NPC cells after glycan digestion by peptide-N-glycosidase (PNGase). B. Flow cytometry on DC-SIGNbound NPC cells showing no interference of fucose at $20 \mathrm{mM}$. C. Flow cytometry on DC-SIGN-bound NPC cells showing dose-dependent inhibition by mannose (left panel). Regression plot suggested the $50 \%$ inhibition concentration $\left(\mathrm{IC}_{50}\right)$ at $10 \mathrm{mM}$ (right panel). Three independent experiments were performed. 
bound by ANXA2 on NPC cells. In addition, ANXA2 can be distributed in a variety of cellular compartments including cytoplasm, intracellular membranes and external face of the plasma membrane [27]. The reduction of ANXA2 by RNA interference may not decrease its distribution on the cell surface as much as inside the cell. Indeed, another study on hepatoma xenograft in mice demonstrated that the distribution of ANXA2 is mainly localized to the cell membrane in shRNA-bearing group [28]. The binding capacity of DC-SIGN by ANXA2 could be underestimated.

It is striking that the ANXA2-knockdown NPC cells were significantly reduced in promoting IL-10 production in DCs (Fig. 6C) and suppressed in xenograft growth (Fig. 6D). According to other immunohistochemical studies, IL10 is detected in NPC biopsies [29]. Interestingly, IL-10 is present in the leucocyte infiltrate of the tumor instead of in the NPC cells [30]. Herein our result suggests a substantial involvement of ANXA2 and DC-SIGN interaction in regulating cytokine production of DCs and the progression of NPC. Further studies may be needed to distinguish the effects of exoplasmic from cytoplasmic ANXA2 on tumor growth.

IL-10 is a cytokine associated with immune suppression. It was identified as an inhibitor for other cytokine synthesis and antigen presentation [31]. Inhibitory effect of IL-10 on T cells was mediated mainly through APCs such as DCs [8]. High concentrations of IL-10 promote naïve $\mathrm{T}$ cells to differentiate into regulatory $\mathrm{T}$ (Treg) cells [32], and Treg cells produce more IL-10 in positive feedback regulation [33]. These mechanisms decrease production of pro-inflammation helper $\mathrm{T}$ cells to inhibit immune responses. It seems likely that cancer cells, including NPC cells, commonly regulate immune suppression by stimulating IL-10 production in DCs. Supporting evidence was demonstrated in the present study. We used LPS to activate Toll-like receptor pathway which induced both IL-12 and IL-10 production in MDDCs [7] (Fig. 1B); however, when co-cultured with NPC cells, MDDCs increased IL-10 but decreased IL-12 production, supporting an immunosuppressive response.

Other molecules may be involved in the interactions of ANXA2 and DC-SIGN. An example is C1q, an ANXA2 receptor initiating the classical complement pathway to facilitate phagocytosis [34] and a DC-SIGN partner modulating the differentiation of MDDCs [35]. Future works may be required for us to reveal more details of these interactions and provide practicable advice in cancer immunotherapy.

\section{MATERIALS AND METHODS}

\begin{abstract}
Animals
To monitor the NPC growth in vivo, immunodeficient NOD/SCID/IL2r- $\gamma$ null (NSG) mice were engrafted with TW01 NPC cell lines. A total of $2 \times 10^{7}$ cells in $100 \mu \mathrm{l}$ of PBS was transplanted subcutaneously (s.c.) in the mouse abdomen. After 14 days, the tumor size was measured by Short*Short*Long/2 $\left(\mathrm{mm}^{3}\right)$. Totally 7 mice in each group were examined. All the animal procedures were approved by the Laboratory Animal Center in Taipei Medical University.
\end{abstract}

\section{Preparation of cells}

NPC cell line TW01 is Epstein-Barr virus negative and derived from the NPC of a Taiwanese patient [36]. It is categorized as WHO type I, squamous cell carcinoma. The cells were maintained in DMEM containing 10\% FBS, 100 units $/ \mathrm{ml}$ penicillin, and $100 \mu \mathrm{g} / \mathrm{ml}$ streptomycin at $37^{\circ} \mathrm{C}$ with $5 \% \mathrm{CO}_{2}$.

Monocyte-derived DCs (MDDCs) were prepared from primary monocytes $\left(\mathrm{CD} 14^{+}\right)$separated from the peripheral blood mononuclear cells of healthy donors by magnetic beads conjugated with anti-CD14 antibody (MACS, Germany). Totally nine healthy subjects were recruited in the study. Primary cells were maintained in RPMI 1640 medium containing 10\% FBS (Biological Industries, Israel), 100 units $/ \mathrm{ml}$ penicillin, and $100 \mu \mathrm{g} /$ $\mathrm{ml}$ streptomycin at $37^{\circ} \mathrm{C}$ with $5 \% \mathrm{CO}_{2}$. Monocytes were cultured in a medium containing IL-4 $(25 \mathrm{ng} / \mathrm{ml})$ and granulocyte monocyte colony-stimulating factor $(50 \mathrm{ng} /$ $\mathrm{ml}$ ) for six to seven days to be differentiated into immature MDDCs. The cultured MDDCs were over 90\% survival rate and purity to be used in the following experiments. All the procedures involving human samples were approved by Taipei Medical University-Joint Institutional Review Board.

\section{Antibodies}

Anti-CD11c-FITC, anti-HLA-DR-FITC, antiCD-86-PE, anti-DC-SIGN-PE, and anti-CD14-PE were purchased for flow cytometry from eBioscience (San Diego, CA, USA). Anti-DC-SIGN antibody used to block the DC-SIGN receptor was from R\&D system (Minneapolis, MN, USA). Anti-ANXA2 antibody was from R\&D system or GeneTex (Irvine, CA, USA). Alkaline phosphatase-conjugated goat anti-mouse IgG and goat anti-rabbit IgG were from Bioscience (Taipei, Taiwan, ROC). Human IL-10 and IL-12 antibodies were from PeproTech. Rabbit IgG $(\mathrm{H}+\mathrm{L})$ reabsorbed secondary 
antibody (conjugated DyLight 594) and anti-human IgG (Fc fragment) antibody (conjugated FITC or HRP) were from GeneTex.

\section{Flow cytometry}

To analyze the DC maturation marker HLA-DR and DC-SIGN, we cultured MDDCs at $5 \times 10^{4}$ per well and stimulated them with $5 \times 105$ NPC cells. After $24 \mathrm{~h}$, we collected MDDCs and NPC cells together and washed with PBS containing $1 \%$ BSA. They were subsequently incubated with anti-human CD11c-PE antibody (GeneTex) and anti-human HLA-DR-FITC or DC-SIGN-FITC antibody (GeneTex) at $4^{\circ} \mathrm{C}$ for $1 \mathrm{~h}$, and then washed twice with PBS containing 1\% BSA. The stained cells were analyzed with FACSCalibur to determine the number of CD11c and HLA-DR or DC-SIGN double positive cells. Data were processed with CellQuest software.

To detect the DC-SIGN ligand expression on NPC cell membranes, $2 \times 10^{5}$ NPC cells were collected and washed with PBS containing $0.1 \%$ FBS. They were subsequently incubated with the recombinant protein consisting of the extracellular domain of DC-SIGN and the $\mathrm{Fc}$-domain of human IgG1 (DC-SIGN-Fc) recombinant protein (R\&D system, USA) at $10 \mu \mathrm{g} / \mathrm{ml}$ for $1 \mathrm{~h}$ at room temperature. After incubation, we detected the DC-SIGNFc recombinant protein on NPCs with an anti-DC-SIGN antibody.

To determine the major DC-SIGN glycan ligand on NPC cells, $4 \times 10^{5}$ NPC cells were collected and washed with PBS containing 1\% BSA. They were subsequently incubated with $2.5 \mu \mathrm{g} / \mathrm{ml}$ DC-SIGN-Fc protein, $10 \mathrm{mM}$ $\mathrm{CaCl}_{2}$ and several concentrations $(0,5,10,15,20,30$, $50,70 \mathrm{mM}$ ) of monosaccharide including mannose and fucose for $1 \mathrm{~h}$ at $4^{\circ} \mathrm{C}$. After incubation, we detected the DC-SIGN-Fc bound on NPCs with anti-Fc-FITC antibody (GeneTex).

To confirm ANXA2 as a DC-SIGN ligand on NPC cells, $4 \times 10^{5}$ NPC cells treated with scramble shRNA (scNPC) or an ANXA2 shRNA (shNPC-1 or shNPC-2) were collected and washed with PBS containing $1 \% \mathrm{BSA}$. The cells were subsequently incubated with $2.5 \mu \mathrm{g} / \mathrm{ml}$ DCSIGN-Fc and $10 \mathrm{mM} \mathrm{CaCl}_{2}$ at $4{ }^{\circ} \mathrm{C}$. After incubation, we detected the DC-SIGN-Fc on NPC cells with anti-Fc-FITC antibody.

\section{Enzyme-linked immunosorbent assay (ELISA)}

Anti-human IL-10 or IL-12 antibody (Invitrogen, USA) was diluted in Coating Buffer A (Invitrogen, USA) to a concentration of $1 \mu \mathrm{g} / \mathrm{ml}$ and used for the detection of NPC mediated IL-10 or IL-12 release from MDDCs. MDDCs were cultured in 24-well plates at a density of $5 \times 10^{4}$ cells per well. In the DC-SIGN blockage analysis, the MDDCs were pre-treated with $1 \mu \mathrm{g} / \mathrm{ml}$ Anti-DC-SIGN antibody (R\&D system, USA) for $1 \mathrm{~h}$. They were then stimulated with $5 \times 10^{5}$ NPC cells. In ANXA2 blockage analysis, NPC cells were pre-treated with $3 \mu \mathrm{g} / \mathrm{ml}$ Antiannexin A2 antibody (R\&D system, USA) for $1 \mathrm{~h}$ before the co-culture.

As suggested in the protocol, $100 \mu \mathrm{l}$ of diluted antihuman IL-10 or IL-12 antibody was added to individual wells of ELISA plates at $4^{\circ} \mathrm{C}$ overnight. The capture antibody-coated plates were washed and blocked with Assay Buffer. For comparison, serial diluted standards were added into corresponding wells of microtiter plate along with $100 \mu \mathrm{l}$ of supernatant from the co-culture and $0.16 \mu \mathrm{g} / \mathrm{ml}$ detecting antibodies. After $2 \mathrm{~h}$ of incubation at room temperature, the plate was washed followed by additional diluted avidin-HRP conjugate and incubated for $30 \mathrm{~min}$ in room temperature. At the end, Stop Solution was added and the reaction was sit for $30 \mathrm{~min}$ in room temperature. Color of each reaction was developed following substrate incubation and measured by Microtiter plate reader at $450 \mathrm{~nm}$ with wavelength correction set at $650 \mathrm{~nm}$. To minimize the effects of variation among individual donors, the data were normalized in each experiment as indicated.

\section{Immunofluorescent staining}

NPC cells $\left(10^{4}\right)$ were seeded on Chamber slides for 2 days. Cells were fixed with $4 \%$ paraformaldehyde for 15 min and blocked with $2 \%$ BSA on ice for $1 \mathrm{~h}$ after washing. DC-SIGN-Fc recombinant protein or IgG at 10 $\mu \mathrm{g} / \mathrm{ml}$ was incubated with anti-ANXA2 antibody (1:100 in 1X TBS) in the presence of $10 \mathrm{mM} \mathrm{Ca}^{2+}$ or $20 \mathrm{mM}$ EDTA at room temperature for $1 \mathrm{~h}$. Anti-Human $\operatorname{IgG}(\mathrm{Fc}$ fragment) antibody-FITC or anti-rabbit IgG antibodyDyLight 594 (1:100 in 1X TBS) was added at room temperature for $1 \mathrm{~h}$ after washing. Samples were mounted with DAPI at least $30 \mathrm{~min}$. Fluorescence imaging was performed using TCS SP5 Confocal Spectral Microscope Imaging System (Leica, Germany).

\section{Immunohistochemistry}

Formalin-fixed and paraffin-embedded human tissues from three NPC subjects were sectioned in $5 \mu \mathrm{m}$ and deparaffinized in xylene. Sections were incubated at $120^{\circ} \mathrm{C}$ in $10 \mathrm{mM}$ citric acid, $\mathrm{pH} 6$ for $20 \mathrm{~min}$ for antigen retrieval. Anti-ANXA2 monoclonal antibody (R\&D) was applied followed by HRP-conjugated secondary antibody incubation. The sections were developed using 3,3'-Diamino-benzidine hydrochloride (DAB). Counterstaining was performed with hematoxylin before dehydration and mounting. All the procedures involving human samples were approved by Taipei Medical University-Joint Institutional Review Board. 


\section{RNA interference}

DC-SIGN siRNA (5'-GGC AAU GGC UGG AAC GAC GAC AAA U-3') was added into the MDDC culture at day 1 . The transfected MDDCs at $2 \times 10^{4}$ per well were seeded on a 24 -well plate and co-cultured with $2 \times 10^{5} \mathrm{NPC}$ for $24 \mathrm{~h}$. The co-cultured media were analyzed for IL-10 concentration.

ANXA2 lentiviral shRNAs (TRCN0000289717 or \#1, 5'-GCAGGAAATTAACAGAGTCTA-3' and TRCN0000289781 or \#2, 5'-CGGGATGCTTTGAACATTGAA-3') and scramble shRNA control (pLAS.Void) with mismatch sequences were purchased from Academia Sinica of Taiwan. They were used for transfection of the packaging HEK 293T cells with helper vectors, using Fugene 6 transfection reagent (Roche). The medium containing lentiviral particles was harvested, filtered, aliquoted and stored at $-80^{\circ} \mathrm{C}$. These viruses were used to transduce $5 \times 10^{5} \mathrm{NPC}$ cells/well in the presence of $8 \mu \mathrm{g} / \mathrm{ml}$ polybrene (SigmaAldrich) in a 24-well plate. Transduced cells were selected in DMEM medium containing $5 \mu \mathrm{g} / \mathrm{ml}$ puromycin (SigmaAldrich).

\section{Preparation of NPC membrane proteins}

To prepare NPC membrane proteins, cells at $3 \sim 5 \times 10^{6}$ from a $10-\mathrm{cm}$ dish were washed and incubated with the extraction buffer 1 containing protease inhibitor (membrane protein extraction kit) at $4^{\circ} \mathrm{C}$ for $15 \mathrm{~min}$. After removing the buffer 1 , the cells were incubated with the extraction buffer 2 at $4^{\circ} \mathrm{C}$ for $30 \mathrm{~min}$. Finally, we collected the supernatant which was enriched in membrane proteins.

\section{Co-immunoprecipitation}

Briefly, $2 \mu \mathrm{g}$ of DC-SIGN-Fc recombinant protein was preincubated in $100 \mu \mathrm{l}$ of protein A magnetic beads with continuous end-to-end gentle rotation at $4^{\circ} \mathrm{C}$ for $2 \mathrm{~h}$. At the end of reaction, magnetic beads were washed with TBST. For precipitation, NPC membrane proteins in the presence of $\mathrm{CaCl}_{2}$ or EDTA (as control) were incubated with beads pre-bound DC-SIGN-Fc with continuous end-to-end gentle rotation at $4^{\circ} \mathrm{C}$ for $2 \mathrm{~h}$. At the end of precipitation, magnetic beads containing complexes of DC-SIGN-Fc and its binding components were eluted by $0.2 \mathrm{M}$ glycine $(\mathrm{pH} \mathrm{2.5)}$ to separate the protein from the beads and neutralized in $1 \mathrm{M}$ Tris $(\mathrm{pH} 8.5)$. We analyzed the eluted proteins using SDS-PAGE and silver staining (Bio-Rad, USA).

\section{LC/MS/MS analysis}

All mass spectrometric analyses were performed using an LTQ-Orbitrap (Discovery) hybrid mass spectrometer with a nanoelectrospray ionization source (ThermoElectron, San Jose, CA, USA) coupled to a nanoflow high-performance liquid chromatography (HPLC) system (Agilent Technologies 1200 series, Germany). An Agilent C18 column (100_0.075mm, 3.5 mm particle diameter) with mobile phases of $\mathrm{A}(0.1 \%$ formic acid in water) and $\mathrm{B}(0.1 \%$ formic acid in acetonitrile) was used. The pump flow rate was set at $0.5 \mathrm{~mL} / \mathrm{min}$, and peptide elution was achieved using a linear gradient of $5-35 \% \mathrm{~B}$ for the first $30 \mathrm{~min}$ followed by a rapid increase to $95 \%$ B over the next $10 \mathrm{~min}$. The conventional MS spectra (Survey Scan) were acquired at high resolution (M/DM, 60,000 full width half maximum) over the acquisition range of $\mathrm{m} / \mathrm{z} 200-2000$ and a series of precursor ions were selected for the MS/MS scan. The former examined the accurate mass and the charge state of the selected precursor ion, while the latter acquired the spectrum (CID spectrum $\mathrm{m}$ or MS/MS spectrum) for the fragment ions generated by collision-induced dissociation.

The mass spectrometry dataset was analyzed using Xcalibur software (version 2.0 SR1). Product ion scans obtained from the MS/MS experiments were investigated using the database search software SEQUEST (TURBO).

\section{Glycan digestion assay}

For peptide-N-glycosidase (PNGase) F (New England Biolabs, USA) treatment, membrane proteins $(180 \mu \mathrm{g})$ were prepared from NPC cells and suspended in $25 \mathrm{mM}$ Tris and $150 \mathrm{mM} \mathrm{NaCl}$ with or without $50,000 \mathrm{U} /$ $\mathrm{ml}$ PNGase $\mathrm{F}$ at $37^{\circ} \mathrm{C}$ for $2 \mathrm{~h}$. After digestion membrane proteins were precipitated with DC-SIGN-Fc, followed by SDS-PAGE and immunoblotting with anti-annexin A2 or anti-DC-SIGN antibody.

\section{Reverse transcriptase quantitative polymerase chain reaction (RT-qPCR)}

Total RNAs $(1 \mu \mathrm{g})$ were reversely transcribed using oligo-dT primers and GoScript ${ }^{\mathrm{TM}}$ Reverse Transcription System (Promega, life sciences). Quantitative PCR was


LightCycler ${ }^{\circledR} 480$ (Roche) according to the manufacturer's protocol. The PCR samples were incubated $1 \mathrm{~min}$ at $50^{\circ} \mathrm{C}$ followed by $1 \mathrm{~min}$ at $95^{\circ} \mathrm{C}$ and 40 cycles of $5 \mathrm{~s}$ at $95^{\circ} \mathrm{C}$ and $30 \mathrm{~s}$ at $60^{\circ} \mathrm{C}$. The dissociation reaction was performed as $1 \mathrm{~min}$ at $95^{\circ} \mathrm{C}$ and $30 \mathrm{~s}$ at $55^{\circ} \mathrm{C}$. The primer sequences used are 5'-CTCTACACCCCCAAGTGCAT-3' (Forward) and 5'-TCAGTGCTGATGCAAGTTCC-3' (Reverse) for ANXA2; 5'-CAGCAAGAGCACAAGAGGAAG-3' 
(Forward) and 5'-TGGTACATGACAAGGTGCGG-3' (Reverse) for glyceraldehyde 3-phosphate dehydrogenase (GAPDH) as a control.

\section{Western blotting}

Proteins from SDS-PAGE were transferred to PVDF membranes at $100 \mathrm{~V} / 400 \mathrm{~mA}$ for $2 \mathrm{~h}$, and blocked at room temperature for $1 \mathrm{~h}$. After blocking, the membrane was incubated in $1 \% \mathrm{BSA} / \mathrm{PBS}$ with the anti-annexin A2 (R\&D system, USA) or anti-DC-SIGN (GeneTex, USA) primary antibody in a 1:5,000 dilution with gentle shaking at $4^{\circ} \mathrm{C}$ overnight. After washed for three times with PBST, the membrane was incubated with the HRP-conjugated anti-mouse (GeneTex) or rabbit IgG (GeneTex) secondary antibody in a 1:10,000 dilution for $2 \mathrm{~h}$. After the secondary antibody incubation, the membrane was washed 3 times with PBST. For visualization, the membrane was treated with Super-Signal West Pico Chemiluminescent kit (Thermo Fisher Scientific) and imaged.

\section{Statistical methods}

Each experiment was performed at least three times. SigmaPlot software and the Student $t$ test were used for statistical analysis. A value of $p<0.05$ was considered statistically significant. Results are shown as mean \pm standard deviation.

\section{ACKNOWLEDGEMENTS}

The authors thank Dr. Ching-Hwa Tsai in the Graduate Institute of Microbiology, National Taiwan University for the gift of NPC TW01 cell line, and Dr. Shauh-Der Yeh in the Department of Urology, Taipei Medical University Hospital for his helps on animal experiments. We also thank Shih-Han Tsai, ChengFan Lee and Yu-Hsuan Sung in the School of Medical Laboratory Science and Biotechnology, Taipei Medical University for their helps on experiment preparations. This work was financially supported by Taipei Medical University-Shuang-Ho Hospital (103TMU-SHH-22) and Ministry of Science and Technology in Taiwan (NSC 1012320-B-038-028 and NSC 102-2320-B-038-033).

\section{Author Contributions}

PZC, MSH, YFL and CHC designed research. PZC, TJH, YTL and CHL performed research. MSH, CWC, CCL, TKL, FPL, YFL and CHC analyzed data. CWC, YFL and CHC wrote paper.

\section{Conflict of Interest Disclosures}

All the authors declare no conflict of interest on the publication.

\section{REFERENCES}

1. Radford KJ, Tullett KM and Lahoud MH. Dendritic cells and cancer immunotherapy. Curr Opin Immunol. 2014; 27:26-32.

2. Lanzavecchia A and Sallusto F. Regulation of $\mathrm{T}$ cell immunity by dendritic cells. Cell. 2001; 106(3):263-266.

3. Geijtenbeek TB, van Vliet SJ, Engering A, t Hart BA and van Kooyk Y. Self- and nonself-recognition by C-type lectins on dendritic cells. Annu Rev Immunol. 2004; 22:3354.

4. Geijtenbeek TB, Kwon DS, Torensma R, van Vliet SJ, van Duijnhoven GC, Middel J, Cornelissen IL, Nottet HS, KewalRamani VN, Littman DR, Figdor CG and van Kooyk Y. DC-SIGN, a dendritic cell-specific HIV-1-binding protein that enhances trans-infection of T cells. Cell. 2000; 100(5):587-597.

5. den Dunnen J, Gringhuis SI and Geijtenbeek TB. Innate signaling by the C-type lectin DC-SIGN dictates immune responses. Cancer immunology, immunotherapy : CII. 2009; 58(7):1149-1157.

6. Hsu SC, Chen CH, Tsai SH, Kawasaki H, Hung CH, Chu YT, Chang HW, Zhou Y, Fu J, Plunkett B, Su SN, Vieths S, Lee RT, Lee YC and Huang SK. Functional interaction of common allergens and a C-type lectin receptor, dendritic cell-specific ICAM3-grabbing non-integrin (DC-SIGN), on human dendritic cells. J Biol Chem. 2010; 285(11):79037910 .

7. Svajger U, Anderluh M, Jeras M and Obermajer N. C-type lectin DC-SIGN: an adhesion, signalling and antigen-uptake molecule that guides dendritic cells in immunity. Cell Signal. 2010; 22(10):1397-1405.

8. Grutz G. New insights into the molecular mechanism of interleukin-10-mediated immunosuppression. J Leukoc Biol. 2005; 77(1):3-15.

9. Vicari AP, Caux C and Trinchieri G. Tumour escape from immune surveillance through dendritic cell inactivation. Seminars in cancer biology. 2002; 12(1):33-42.

10. Aarnoudse CA, Garcia Vallejo JJ, Saeland E and van Kooyk Y. Recognition of tumor glycans by antigen-presenting cells. Curr Opin Immunol. 2006; 18(1):105-111.

11. Gringhuis SI, den Dunnen J, Litjens M, van der Vlist M and Geijtenbeek TB. Carbohydrate-specific signaling through the DC-SIGN signalosome tailors immunity to Mycobacterium tuberculosis, HIV-1 and Helicobacter pylori. Nat Immunol. 2009; 10(10):1081-1088.

12. Saeland E, Belo AI, Mongera S, van Die I, Meijer GA and van Kooyk Y. Differential glycosylation of MUC1 and CEACAM5 between normal mucosa and tumour tissue 
of colon cancer patients. International journal of cancer Journal international du cancer. 2012; 131(1):117-128.

13. Nonaka M, Ma BY, Imaeda H, Kawabe K, Kawasaki N, Hodohara K, Kawasaki N, Andoh A, Fujiyama Y and Kawasaki T. Dendritic cell-specific intercellular adhesion molecule 3-grabbing non-integrin (DC-SIGN) recognizes a novel ligand, Mac-2-binding protein, characteristically expressed on human colorectal carcinomas. J Biol Chem. 2011; 286(25):22403-22413.

14. van Gisbergen KP, Aarnoudse CA, Meijer GA, Geijtenbeek TB and van Kooyk Y. Dendritic cells recognize tumorspecific glycosylation of carcinoembryonic antigen on colorectal cancer cells through dendritic cell-specific intercellular adhesion molecule-3-grabbing nonintegrin. Cancer Res. 2005; 65(13):5935-5944.

15. van Kooyk Y and Geijtenbeek TB. DC-SIGN: escape mechanism for pathogens. Nat Rev Immunol. 2003; 3(9):697-709.

16. Xu T, Tang J, Gu M, Liu L, Wei W and Yang H. Recurrent nasopharyngeal carcinoma: a clinical dilemma and challenge. Current oncology. 2013; 20(5):e406-419.

17. Giannini A, Bianchi S, Messerini L, Gallo O, Gallina E, Asprella Libonati G, Olmi P and Zampi G. Prognostic significance of accessory cells and lymphocytes in nasopharyngeal carcinoma. Pathol Res Pract. 1991; 187(4):496-502.

18. Zong YS, Zhang CQ, Zhang F, Ruan JB, Chen MY, Feng $\mathrm{KT}$ and Yu ZF. Infiltrating lymphocytes and accessory cells in nasopharyngeal carcinoma. Japanese journal of cancer research : Gann. 1993; 84(8):900-905.

19. Hartmann E, Wollenberg B, Rothenfusser S, Wagner M, Wellisch D, Mack B, Giese T, Gires O, Endres S and Hartmann G. Identification and functional analysis of tumor-infiltrating plasmacytoid dendritic cells in head and neck cancer. Cancer Res. 2003; 63(19):6478-6487.

20. Xu YF, Liu WL, Dong JQ, Liu WS, Feng QS, Chen LZ, Zeng YX, Zeng MS and Jia WH. Sequencing of DCSIGN promoter indicates an association between promoter variation and risk of nasopharyngeal carcinoma in cantonese. BMC Med Genet. 2010; 11:161.

21. Waisman DM. Annexin II tetramer: structure and function. Mol Cell Biochem. 1995; 149-150:301-322.

22. Bharadwaj A, Bydoun M, Holloway R and Waisman D. Annexin A2 heterotetramer: structure and function. International journal of molecular sciences. 2013; 14(3):6259-6305.

23. Zhang X, Liu S, Guo C, Zong J and Sun MZ. The association of annexin A2 and cancers. Clin Transl Oncol. 2012; 14(9):634-640.

24. Goulet F, Moore KG and Sartorelli AC. Glycosylation of annexin I and annexin II. Biochem Biophys Res Commun. 1992; 188(2):554-558.

25. Woodham AW, Raff AB, Raff LM, Da Silva DM, Yan L, Skeate JG, Wong MK, Lin YG and Kast WM. Inhibition of Langerhans cell maturation by human papillomavirus type 16: a novel role for the annexin A2 heterotetramer in immune suppression. J Immunol. 2014; 192(10):4748-4757.

26. Nonaka M, Ma BY, Murai R, Nakamura N, Baba M, Kawasaki N, Hodohara K, Asano S and Kawasaki T. Glycosylation-Dependent Interactions of C-Type Lectin DC-SIGN with Colorectal Tumor-Associated Lewis Glycans Impair the Function and Differentiation of Monocyte-Derived Dendritic Cells. The Journal of Immunology. 2008; 180(5):3347-3356.

27. Luo M and Hajjar KA. Annexin A2 system in human biology: cell surface and beyond. Seminars in thrombosis and hemostasis. 2013; 39(4):338-346.

28. Dong Z, Yao M, Zhang H, Wang L, Huang H, Yan $\mathrm{M}, \mathrm{Wu} \mathrm{W}$ and Yao D. Inhibition of Annexin A2 gene transcription is a promising molecular target for hepatoma cell proliferation and metastasis. Oncology letters. 2014; 7(1):28-34.

29. Ozyar E, Ayhan A, Korcum AF and Atahan IL. Prognostic role of Ebstein-Barr virus latent membrane protein-1 and interleukin-10 expression in patients with nasopharyngeal carcinoma. Cancer investigation. 2004; 22(4):483-491.

30. Beck A, Pazolt D, Grabenbauer GG, Nicholls JM, Herbst H, Young LS and Niedobitek G. Expression of cytokine and chemokine genes in Epstein-Barr virus-associated nasopharyngeal carcinoma: comparison with Hodgkin's disease. The Journal of pathology. 2001; 194(2):145-151.

31. Fiorentino DF, Bond MW and Mosmann TR. Two types of mouse T helper cell. IV. Th2 clones secrete a factor that inhibits cytokine production by Th1 clones. J Exp Med. 1989; 170(6):2081-2095.

32. Heidt S, Segundo DS, Chadha R and Wood KJ. The impact of Th17 cells on transplant rejection and the induction of tolerance. Curr Opin Organ Transplant. 2010; 15(4):456461.

33. Chang J, Kunkel SL and Chang $\mathrm{CH}$. Negative regulation of MyD88-dependent signaling by IL-10 in dendritic cells. Proc Natl Acad Sci U S A. 2009; 106(43):18327-18332.

34. Martin M, Leffler J and Blom AM. Annexin A2 and A5 serve as new ligands for $\mathrm{C} 1 \mathrm{q}$ on apoptotic cells. J Biol Chem. 2012; 287(40):33733-33744.

35. Hosszu KK, Valentino A, Vinayagasundaram U, Vinayagasundaram R, Joyce MG, Ji Y, Peerschke EI and Ghebrehiwet B. DC-SIGN, C1q, and gC1qR form a trimolecular receptor complex on the surface of monocytederived immature dendritic cells. Blood. 2012; 120(6):12281236.

36. Lin CT, Wong CI, Chan WY, Tzung KW, Ho JK, Hsu $\mathrm{MM}$ and Chuang SM. Establishment and characterization of two nasopharyngeal carcinoma cell lines. Laboratory investigation; a journal of technical methods and pathology. 1990; 62(6):713-724. 